\title{
Optimization of unmanned aerial vehicle augmented ultra-dense networks
}

\author{
Alireza Zamani ${ }^{1}$, Robert Kämmer ${ }^{1}$, Yulin $\mathrm{Hu}^{2^{*}}$ (D) and Anke Schmeink ${ }^{1}$
}

\author{
*Correspondence: \\ hu@umic.rwth-aachen.de \\ ${ }^{2}$ Informationstheorie und \\ Systematischer Entwurf von \\ Kommunikationssystemen, RWTH \\ Aachen University, Kopernikusstr. \\ 16, 52074, Aachen, Germany \\ Full list of author information is \\ available at the end of the article
}

\begin{abstract}
In this paper, we study the integration of unmanned aerial vehicle small cells (UAV-SCS) for the purpose of augmenting or temporarily restoring service to an ultra-dense cellular network. The aim is to minimize the overall power consumption of the network by jointly optimizing the number of UAV-SCs, their placement, associations, and the power allocation, subject to user QoS (quality of service), transmit power, and fronthaul capacity constraints. As the resulting optimization problem is non-convex and computationally inefficient to solve, we investigate lower complexity alternatives. By reformulating the original problem, a linear structure can be obtained that is efficiently solved by off-the-shelf solvers. Furthermore, we also propose a meta-heuristic method that is based on particle swarm optimization. The performance of the proposed methods is evaluated via simulation studies and compared to state-of-the-art techniques. The results illustrate that the proposed methods consistently outperform conventional techniques by deploying fewer UAV-SCs and also lowering the transmit powers. Furthermore, considerable power savings were observed particularly for low QoS demands and dense scenarios.
\end{abstract}

Keywords: Ultra-dense cellular networks, Unmanned aerial vehicle small cells, Resource allocation, User association, UAV placement

\section{Introduction}

The use of UAVs in communication networks, as aerial base stations or relays, is justified by their strong line of sight links, the possibility of rapid deployment, and modest costs. For this purpose, low altitude platforms, e.g., rotary-wing UAVs, can be used for network augmentation or alleviating high traffic by hovering at the deployed position for several hours [1]. However, the integration of UAV-SCs into communication networks and their coexistence faces many open challenges. Having full control on the 3D mobility of UAVs raises the question of their placement. The authors in [2], developed a unified softwaredefined framework for matching dynamic traffic demands using moving base stations. In addition to the positioning of UAV-SCs, the optimization of user associations and power control is also critical [3, 4]. This is because the associations between user demand nodes and access points have a strong influence on the resources available for allocation [5], e.g., power and fronthaul capacity. This issue becomes particularly relevant to an interferencelimited ultra-dense network operating with limited capacity fronthaul links.

(c) The Author(s). 2020 Open Access This article is licensed under a Creative Commons Attribution 4.0 International License, which permits use, sharing, adaptation, distribution and reproduction in any medium or format, as long as you give appropriate credit to the original author(s) and the source, provide a link to the Creative Commons licence, and indicate if changes were made. The images or other third party material in this article are included in the article's Creative Commons licence, unless indicated otherwise in a credit line to the material. If material is not included in the article's Creative Commons licence and your intended use is not permitted by statutory regulation or exceeds the permitted use, you will need to obtain permission directly from the copyright holder. To view a copy of this licence, visit http://creativecommons.org/licenses/by/4.0/. 
Prior works [6-9] in literature have studied the user association optimization and UAV-base station positioning, with fixed transmit power and negligible interference. This problem is generally approached by finding the minimum number of UAVs that will ensure non-overlapping coverage. The work in [10] similarly investigated the deployment of a single UAV, while considering negligible interference and given user associations. Reference [11] considered a stationary low altitude platform and used circle packing for providing maximum coverage at minimum cost. The authors in [12] proposed an MINLP for the placement of a single UAV, subject to user signal-tonoise ratio requirements. The original problem is decomposed to a coverage radius solver, which aims to maximize coverage and a $2 \mathrm{D}$ placement problem that finds the optimal 2D position for a fixed height by solving a mixed integer quadratic program. However, the authors assumed orthogonal users and fixed power allocation. The authors in [13] considered user mobility in a UAV augmented network and applied Q-learning to determine the UAV position and user associations. Note that the aforementioned works offer no insight on the optimal number of UAVs and power allocation.

In contrast to the aforementioned works, joint power and sub-carrier allocation were studied in [14], although no association or UAV positioning is considered. More relevant works, such as [15], find the user associations, bandwidth allocations, and 3D placement via a particle swarm optimization (PSO). Chang et al. [16] addresses user association, trajectory design and power control via reinforcement learning. Most notably, [17] considered UAV-base station placement and transmit power allocation, subject to satisfying the demand of users. The interference was avoided by assuming orthogonal users, and the resulting problem is solved via transport theory. Furthermore, the coexistence of UAVbase stations with an existing cellular network is not considered and the limited capacity of fronthaul links is also ignored.

In this paper, we consider the augmentation of an ultra-dense centralized radio access network (C-RAN) using UAV-SC. The contributions of this work are summarized as follows:

- Different from existing works that assume orthogonal users and interference-free models, we study the coexistence of UAV-SC with ground base stations for the purpose of serving co-channel users. This is practically relevant since in an ultra-dense network with finite spectrum resources and strong line of sight links from the UAV-SCs, achieving orthogonal users may not be possible. Note that studying the system under this assumption implies higher interference levels. Furthermore, this also demands an optimization model that accordingly incorporates the impact of interference depending on the user association and power allocation, which is missing from the literature.

- With the aim of offering an energy-efficient solution, the power consumption is minimized by optimizing the number of UAV-SCs, their placement, associations, and the power allocation subject to demand node QoS requirements, power, and fronthaul capacity constraints. As the resulting problem is non-convex and computationally challenging to solve, we propose lower complexity alternatives. 
- The performance of the proposed techniques is evaluated via simulation studies. The numerical results indicate the considerable power savings are achieved, especially for small cell sizes or low QoS demands.

The remainder of this paper is organized as follows: The UAV augmented C-RAN system model is defined in Section 2. While the power minimization optimization problem is described in Section 3.1, along with the proposed frameworks. In Section 4, the performance of the proposed frameworks is investigated and compared to state-of-the-art solutions. Lastly, Section 5 concludes our work with a brief summary, while Section 6 explains the experiment setup.

\section{System model}

\subsection{C-RAN system model}

We consider the existing ground network to be a C-RAN, where a central unit (CU) supports multiple access points such as remote radio heads (RRHs) and UAV-SC, as shown in Fig. 1. A set of ground co-channel downlink users, defined as $\mathcal{K}=\{1, \ldots, K\}$, populate the network. The average long-term QoS requirement of the $k$ th user is known via the average signal-to-interference-plus-noise ratio (SINR), denoted by $\gamma_{k}$, and equivalent rate requirement (for fixed bandwidth), denoted by $R_{k}$. The set of operational RRHs is defined as $\mathcal{J}=\{1, \ldots, J\}$, each with a maximum transmit power of $P_{j}^{\max }$, where $j$ denotes the index of the $j$ th RRH. We assume that there exists a set of UAVs that can be potentially used for network augmentation, given as $\mathcal{I}=\{1, \ldots, I\}$. Each UAV-SC has a maximum transmit power of $P_{i}^{\max }$, where $i$ denotes the index of the $i$ th UAV-SC. The spatial position of the $i$ th UAV is described by $\left(x_{i}, y_{i}, z_{i}\right)$, which described the longitude, latitude, and altitude, respectively. The association between the $i$ th UAV-SC and the $k$ th user is defined by $\alpha_{i k} \in\{0,1\}$, while the association between the $j$ th $R R H$ and the $k$ th user is described by $\beta_{j k} \in\{0,1\}$. Note that a 1 is used to represent an active connection for both variables. The allocated power to the downlink channel between the $i$ th UAV-SC and the $k$ th UE is denoted by $p_{i k}$, while $p_{j k}$ refers to the allocated power of the corresponding RRH and UE. Note that the relationship between the association and the transmitted power becomes evident, as a user only receives transmission by its associated node.

As the focus of the work is the long-term resource allocation and deployment of UAV-SCs, we consider a time-averaged scenario, neglecting short-term phenomena such as small-scale fading and UAV vibrations. The channel between the RRHs and users is denoted by $h_{j k}$ and consists of LoS and NLoS components. We assume

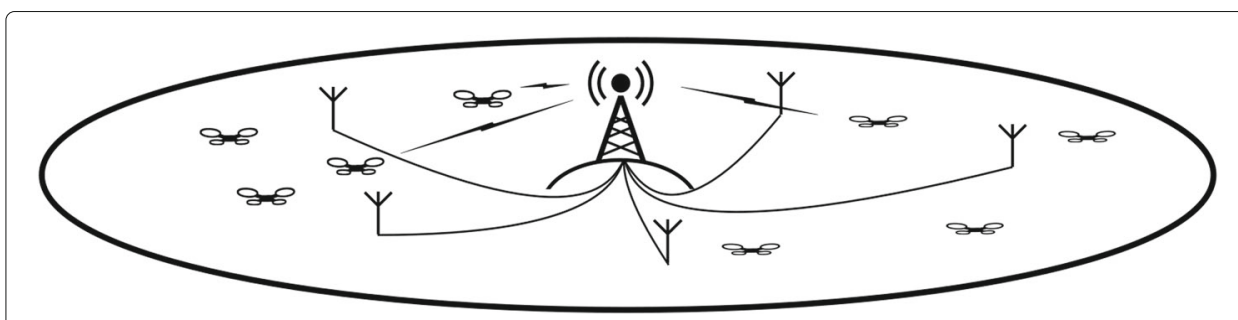

Fig. 1 General network layout of a UAV-SC augmented C-RAN 
that fronthaul connection between the RRHs and the $\mathrm{CU}$ has a finite capacity of $C_{j}$.

\subsection{RRH power consumption model}

The overall RRH power consumption follows [18] and is comprised of a static and a loaddependent component, as follows:

$$
P_{j}^{\mathrm{RRH}}=\left(1-\lambda_{j}\right) P^{\mathrm{idle}}+\lambda_{j} P^{\text {active }}+\Delta \sum_{k} P_{j k},
$$

in which $\Delta$ is the linear load-dependent power gradient, and $\lambda_{j}$ indicates the binary state of the RRH, where 1 indicates active and 0 vice versa. The static power in the active state, represented by $P^{\text {active }}$, accounts for the radio frequency (RF) transceiver chain, the baseband $(\mathrm{BB})$ interface, the direct current (DC) power supply loss, the main supply loss, and the power amplifier (PA) efficiency denoted by $P_{\mathrm{RF}}, P_{\mathrm{BB}}, \mu_{\mathrm{DC}}, \mu_{\mathrm{MS}}$, and $\eta_{\mathrm{PA}}$, respectively. This static power is calculated at the minimum load $P_{\min }=P_{j}^{\max } \times 0.1 \%$ as shown below:

$$
P^{\text {active }}=\frac{\frac{P_{\min }}{\eta_{\mathrm{PA}}}+P_{\mathrm{RF}}+P_{\mathrm{BB}}}{\left(1-\mu_{\mathrm{DC}}\right)\left(1-\mu_{\mathrm{MS}}\right)} .
$$

The values of the aforementioned parameters are summarized in Table 1 . The sum of the power allocated to all the users by a single RRH is subject to the maximum power budget, described as:

$$
\sum_{k} P_{j k} \leq P_{j}^{\max }
$$

\subsection{UAV to ground channel model}

The UAV-SC to user and CU to UAV-SC channels follow a common statistical model from literature $[12,15]$ that is a function of the 3D spatial placement of UAVs, denoted by $h_{i k}\left(x_{i}, y_{i}, h_{i}\right)$ and $h_{i}^{\mathrm{CU}}\left(x_{i}, y_{i}, h_{i}\right)$, respectively. The path loss between the UAV-SC and the ground node is given by:

$$
\mathrm{PL}_{\mathrm{UAV}}=\mathrm{FSPL}+P_{\mathrm{LoS}} \eta_{\mathrm{LOS}}+\left(1-P_{\mathrm{LoS}}\right) \eta_{\mathrm{NLOS}}
$$

where FSPL represents the free space path loss and $\eta_{\mathrm{LoS}}$ and $\eta_{\mathrm{NLoS}}$ denote the additional path loss due to LoS and NLoS as summarized in Table 2. The probability of LoS for the UAV to ground channel depends on the elevation angle, denoted by $\theta$, while the effect of the environment is captured via constants $a$ and $b$ as shown below:

$$
P_{\mathrm{LoS}}=\frac{1}{1+a \exp (-b[\theta-a])} .
$$

Table 1 RRH parameter settings

\begin{tabular}{lll}
\hline Parameter & Symbol & Value \\
\hline RF transceiver power & $P_{\mathrm{RF}}$ & $12.9 \mathrm{~W}$ \\
$\mathrm{BB}$ interface power & $P_{\mathrm{BB}}$ & $29.6 \mathrm{~W}$ \\
$\mathrm{DC}$ power supply loss & $\mu_{\mathrm{DC}}$ & 0.075 \\
MS loss & $\mu_{\mathrm{MS}}$ & 0.09 \\
\hline
\end{tabular}


Table 2 UAV parameter settings

\begin{tabular}{llr}
\hline Parameter & Symbol & Value \\
\hline Total weight & mUAV & $12.9 \mathrm{~kg}$ \\
Rotor radius & $r$ & $0.228 \mathrm{~m}$ \\
Rotor number & $n$ & 6 \\
Hovering power & phover & $936.3 \mathrm{~W}$ \\
\hline
\end{tabular}

\subsection{UAV power consumption model}

For the purpose of UAV-SC placement, the dominant factors of the power consumption consist of the hovering and data transmission, following [19]. The static power consumption relating to the hovering of the UAV is defined as:

$$
P_{\text {hover }}=\sqrt{\frac{(m g)^{3}}{2 \pi r^{2} n \rho}},
$$

where $m, r, n, g$, and $\rho$ denote the UAV weight, rotor radius, rotor number, gravitational constant, and air density, respectively. The values of the aforementioned parameters are summarized in Table 3. The transmission power of the UAV follows a load-dependent model similar to the RRH, with the complete power model described as:

$$
P_{i}^{\mathrm{UAV}}=\mu_{i}\left(P^{\text {hover }}+P^{\text {active }}\right)+\Delta \sum_{k} P_{i k},
$$

where $\mu_{i}$ is a binary variable indicating whether a UAV-SC is deployed and $\Delta$ is the power gradient. Similar to an RRH, the total power allocated by a UAV-SC to the users is subject to a maximum transmit power limit as shown below:

$$
\sum_{k} P_{i k} \leq P_{i}^{\max } .
$$

\subsection{Fronthaul links}

A wired fronthaul link with fixed capacity of $C_{j}$ is assumed for the $j$ th $\mathrm{RRH}$, which leads to the following constraint:

$$
\sum_{k} \alpha_{j k} R_{k} \leq C_{j} .
$$

As the main focus of the work is the integration of UAV-SC, we assume a point to point fronthaul link, with negligible interference on the access network. In practice, this is realized by the advanced capabilities of the $\mathrm{CU}$, which can achieve out-of-band wireless

Table 3 General system parameter settings

\begin{tabular}{ll}
\hline Parameter & Settings \\
\hline Carrier frequency & $2 \mathrm{GHz}$ \\
Bandwidth & $10 \mathrm{MHz}$ \\
Maximum transmit power & $43 \mathrm{dBm}$ \\
RRH fronthaul capacity & $10 \mathrm{Gbps}$ \\
Transmit power gradient & 4.2 \\
LoS path loss & $103.4+24.2 \log _{10} d$ \\
NLoS path loss & $131.1+42.8 \log _{10} d$ \\
PLoS RRH-UE & $\min \left(\frac{0.018}{d}, 1\right)\left(1-\exp \left(-\frac{d}{0.063}\right)\right)$ \\
& $+\exp \left(-\frac{d}{0.063}\right)$ \\
Noise level & $-134 \mathrm{dBm} / \mathrm{Hz}$ \\
\hline
\end{tabular}


fronthaul links or create directive narrow beams. The achievable capacity of the wireless fronthaul links is expressed as:

$$
\sum_{k} \alpha_{i k} R_{k} \leq B W \log _{2}\left(1+\frac{P_{i}^{\mathrm{CU}} h_{i}^{\mathrm{CU}}}{\sigma_{i}^{2}}\right)
$$

where $B W$ and $P_{i}^{\mathrm{CU}}$ are the bandwidth and the power allocated to the wireless fronthaul of the $i$ th UAV-BS, respectively. Without loss of generality, henceforth, it is assumed that both the user rate demand and the achievable capacity are normalized to the respective bandwidths. The $\mathrm{CU}$ is considered to be subject to a transmit power budget:

$$
\sum_{i} P_{i}^{\mathrm{CU}} \leq P_{\text {Tot }}^{\mathrm{CU}}
$$

\subsection{User associations}

In this work, the user association indicates whether a particular user is receiving its data transmission from an access point or not. Therefore, a connected user would incur both a power and a fronthaul cost to the associated access point. Assuming a user can only associate to a single access point, the association is modeled via the following:

$$
\begin{aligned}
& P_{i k} \leq \alpha_{i k} P_{i}^{\max }, \\
& P_{j k} \leq \beta_{j k} P_{j}^{\max }, \\
& \sum_{i} \alpha_{i k}+\sum_{j} \beta_{j k}=1,
\end{aligned}
$$

where (12a) and (12b) relate the associations to the power allocation by ensuring that only an associated user is allocated power, while (12c) guarantees that users connect to a single access point. By this means, the user associations can also be used for defining the state of the UAV-SCs and RRHs as shown below:

$$
\begin{aligned}
\alpha_{i k} & \leq \mu_{i}, \\
\beta_{j k} & \leq \lambda_{j},
\end{aligned}
$$

where $\mu_{i}$ and $\lambda_{j}$ represent the state of UAV-SC and RRH, respectively, with 1 indicating active. Constraints (13a) and (13b) enforce the state of the corresponding access point to be active if there is at least one associated user.

\subsection{Signal model}

The received downlink signal of the $k$ th user, denoted by $z_{k}$, can be described as:

$$
\begin{aligned}
z_{k} & =\sum_{i} \sqrt{P_{i k}} h_{i k} s_{k}+\sum_{j} \sqrt{P_{j k}} h_{j k} s_{k} \\
& +\sum_{i, l \neq k} \sqrt{P_{i l}} h_{i k} s_{l}+\sum_{j, l \neq k} \sqrt{P_{j l}} h_{j k} s_{l}+n_{k},
\end{aligned}
$$

where $s_{k}$ is the data symbol and $n_{k}$ is the additive white Gaussian noise, with zero mean and $\sigma_{k}^{2}$ variance of the $k$ th user. The first and second terms indicate the desired part of the signal from the UAV-SCs and RRHs, respectively. While the third and fourth terms represent the interference from UAV-SCs and RRHs. 


\section{Problem formulation}

In this section, we provide a joint design aiming at minimizing the total power consumption via determining the number of UAV-SC and their hovering locations, the user associations, and the power allocation. In the following, we will first provide the problem formulation and subsequently solve the problem via two approaches. Finally, the complexity levels of the proposed approaches are discussed.

\subsection{Problem formulation}

The power minimization problem is formulated as follows:

P1:

$$
\begin{array}{cl}
\min _{\substack{\alpha_{i k}, \beta_{j k}, \mu_{i}, \lambda_{j}, P_{i k}, P_{j k}, P_{i}^{\mathrm{CU}}, x_{i}, y_{i}, z_{i}}} & \sum_{i} P_{i}^{\mathrm{UAV}}+\sum_{i} P_{i}^{\mathrm{CU}}+\sum_{j} P_{j}^{\mathrm{RRH}} \\
\text { s.t. } & (1),(3),(7),(8),(9),(10),(11), \\
& (12 \mathrm{a}),(12 \mathrm{~b}),(12 \mathrm{c}), \\
& \frac{\sum_{i} P_{i k}\left|h_{i k}\right|^{2}+\sum_{j} P_{j k}\left|h_{j k}\right|^{2}}{\sum_{i, l \neq k} P_{i l}\left|h_{i k}\right|^{2}+\sum_{j, l \neq k} P_{j l}\left|h_{j k}\right|^{2}+\sigma_{k}^{2}} \\
& \geq \gamma_{k}, \\
& P_{i k}, P_{j k}, P_{i}^{\mathrm{CU}} \geq 0, \\
& z_{i} \geq 0, \\
& \alpha_{i k}, \beta_{j k}, \mu_{i}, \lambda_{j} \in\{0,1\}, \\
& i \in \mathcal{I}, j \in \mathcal{J}, k \in \mathcal{K},
\end{array}
$$

where (15a) describes the received SINR of the $k$ th user. Note that the above optimization problem is a mixed integer non-linear programming problem (MINLP), which is NP-hard and computationally expensive to solve. While the binary variable constraints (15d) are non-convex, the major complexity in solving P1 is due to the joint optimization of power allocation and 3D placement in constraint (15a) (which itself is a non-convex function of the 3D placement of a UAV). In practice, powerful off-the-shelf MILP solvers can handle integer constraints efficiently given that they are linear, therefore making convex approximations for the binary variables unnecessary. This motivates the development of an optimization framework with only linear constraints as shown in Section 3.2. The proposed quantization of space and subsequent reformulation is not only motivated by obtaining a linear structure, but it also produces a framework that accommodates the use of channel gains obtained from a radio heat map in the network planning phase. A comparison to low complexity meta-heuristic approaches, such as the one proposed in Section 3.3, provides clearer perspective on the performance.

\subsection{MILP optimization based on quantizing the 3D space}

We introduce a number of candidate UAV locations by quantizing the 3D spatial placement into a set of $N_{1} \times N_{2} \times N_{3}$ discrete candidate locations, where $N_{1}, N_{2}$, and $N_{3}$ 
represent the number discrete points in longitude, latitude, and altitude, respectively ${ }^{1}$. In fact, when the number of candidate locations becomes sufficiently large, the optimal solution to the approximated (quantized placement) problem P2 becomes arbitrarily close to the original problem P1. Hence, the introduction of the discrete candidate locations does not lose the generality of the problem. In practice, there exists a trade-off in choosing the number of candidate locations to balance between complexity and the performance of obtained solution as studied in Section 4.

To this end, a set of candidate locations is introduced as $\mathcal{I}_{\text {cand }}=N_{1} \times N_{2} \times N_{3}$ which contains the quantized fixed locations. By this means, although the variable size is increased, the original problem can be linearized, which can be solved more efficiently, by the following reformulation:

P2:

$$
\begin{aligned}
\min _{\substack{\alpha_{i k}, \beta_{j k}, \mu_{i}, \lambda_{j}, P_{i k}, P_{j k}, P_{i}^{\mathrm{CU}}}} & \sum_{i} P_{i}^{\mathrm{UAV}}+\sum_{i} P_{i}^{\mathrm{CU}}+\sum_{j} P_{j}^{\mathrm{RRH}} \\
\text { s.t. } & (1),(3),(7),(8),(9),(10),(11), \\
& (12 \mathrm{a}),(12 \mathrm{~b}),(12 \mathrm{c}),(15 \mathrm{a}),(13 \mathrm{a}), \\
& (13 \mathrm{~b}),(15 \mathrm{~b}),(15 \mathrm{~d}) \\
& \sum_{i} \mu_{i} \leq I, \\
& i \in \mathcal{I}_{\text {cand }}, j \in \mathcal{J}, k \in \mathcal{K},
\end{aligned}
$$

Observe that in the above optimization problem, the continuous 3D spatial placement is reduced to the candidate set of locations in $\mathcal{I}_{\text {cand }}$. The quantized 3D spatial UAV locations $\left(x_{i}, y_{i}, z_{i}\right)$ are implicitly included, via their statistical channel gains as per Section 2, and the optimal positions are selected by the UAV state indicator, described in Section 2. Furthermore, as the objective aims to minimize the power consumption (comprised of static power that is greater than its transmit power), the optimal solution to P2 also achieves the minimum number of UAV-SCs. Moreover, constraint (16a) ensures that the total number of deployed UAV-SC does not exceed the units available. This reformulation facilitates the conversion of the original MINLP to an MILP, which can now be solved efficiently via state-of-the-art solvers such as Gurobi and MOSEK without any further manipulation.

\subsection{PSO}

Motivated by the strength of meta-heuristic methods in solving complex problems with modest run time, we propose a modified version of the PSO algorithm to solve P2. The aim is to find the best 3D placement for UAVs as well as the power allocation and association that minimizes the overall power expenditure. However, this requires a decoupling of the problem. In order to incorporate binary decision-making for the association, we assume that the user associations are updated after each iteration of the PSO algorithm by using the new locations of the UAVs and selecting the nearest access node. As for finding the optimal transmit powers, a simple power minimization with fixed associations and UAV locations is solved efficiently via water-filling. The algorithm consists of a set of independent particles, denoted by $\mathcal{Q}=\{1, \ldots, Q\}$, which probe the solution space. Each

${ }_{1}^{1}$ In practice, the suitable locations for UAV deployment are usually restricted and can be known in advance, e.g., via radio maps [20]; we also confirm the possibility of considering discrete candidate locations. 
particle has a local state $\mathbf{X}_{q}$, and a velocity of $\mathbf{V}_{q}$, which are updated as it explores the solution space. The particle state essentially refers to the $3 \mathrm{D}$ placement of the access node in continuous space. This approach means that if more than one particle converge to the same location, they can be merged. Additionally, each particle remembers the best local state and the best overall state in terms of the objective reached, denoted by $\mathbf{X}_{q}^{\text {best }}$ and $\mathbf{X}_{\text {all }}^{\text {best }}$, respectively. Note that the objective here is defined as the total power consumption. Therefore, the particles aim to find locations that lead to the minimum power consumption, consistent with the overall objective of the original problem P1. The velocity and the state of the $q$ th particle at the $t$ th iteration are updated accordingly:

$$
\begin{aligned}
& \mathbf{V}_{q}^{t+1} \leftarrow \\
& \mathcal{X}\left(\mathbf{V}_{q}^{t}+\phi_{1}\left(\mathbf{X}_{q}^{\text {best }}-\mathbf{X}_{q}^{t}\right)+\phi_{2}\left(\mathbf{X}_{\text {all }}^{\text {best }}-\mathbf{X}_{q}^{t}\right)\right) \\
& \mathbf{X}_{q}^{t+1} \leftarrow \mathbf{X}_{q}^{t}+\mathbf{V}_{q}^{t+1}
\end{aligned}
$$

where $\mathcal{X}, \phi_{1}, \phi_{2}$ influence the convergence of the algorithm as defined in [21]. We highlight that (1) is different from those in literature, i.e., [15, 22], as it determines not only the placement of the UAV-SC, but also the power allocation to the users. To this end, after finding the associations and deployment positions that maximize a utility function, a power allocation problem subject to QoS and power budget constraints is solved, via water-filling or linear programming. Note that to ensure a suitable implementation of

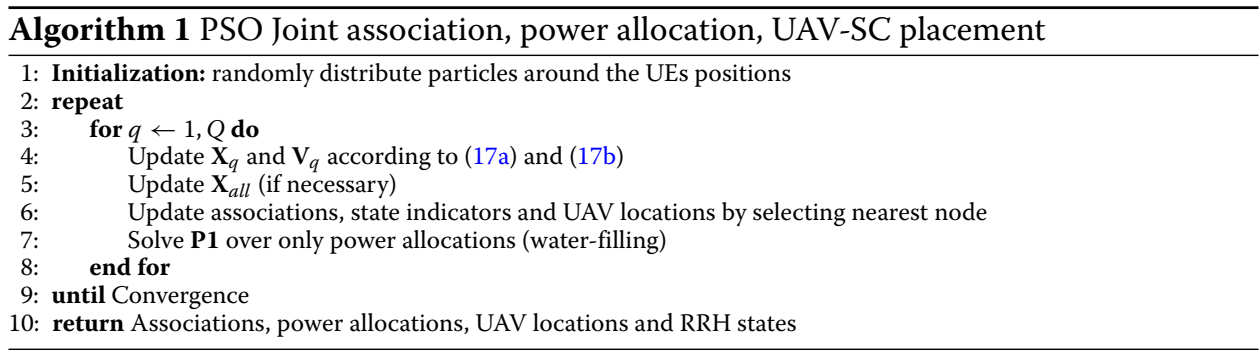

the PSO algorithm, parameters, e.g., the number of particles, were set experimentally as shown in Section 4.

\subsection{Complexity analysis}

As the structure of the original optimization problem is an MINLP, it is typically solved in small sizes via exhaustive search, as the non-linearity makes it incompatible with offthe-shelf solvers. Although P2 remains non-convex, due to the binary variables, the major computational gain is the linear structure that offers compatibility with efficient solvers. In practice, this can be solved by Gurobi in a matter of seconds [23]. Additionally, the complexity now mainly depends on the number of candidate positions or the defined resolution of the grid. Furthermore, we highlight that the framework serves the purpose of network planning which is handled offline with loose time restrictions. Nonetheless, binary approximation techniques, such as [24-26], may be applied to reduce P2 to a linear programming problem with complexity $n^{2} s$, where $n$ is the size of the variables and $s$ is the number of constraints. However, this reduction in complexity comes at the expense of obtaining sub-optimal solutions. The computational complexity and convergence behavior of the proposed PSO algorithm are studied in Section 4. 


\section{Simulation results and discussion}

In this section, the performance of the proposed techniques is studied via Monte Carlo simulations, with results averaged over 200 realizations. The simulation scenarios are set up in accordance with 3GPP LTE specifications, and the simulation parameters are summarized in Tables 1, 2, and 3. Two operational RRHs and six ground downlink users are uniformly distributed in a C-RAN of a radius of $800 \mathrm{~m}$. The 3D grid of 42 candidate UAVSC deployment positions is defined to be equidistant and at heights of $[31,44,57,70]$ meters.

The simulation studies begin with a brief investigation into suitable initialization values for both of the proposed algorithms. We first study the trade-off between power consumption and computation time with increasing quantization points. This helps find a suitable setup for the other simulations. Similarly, the impact of the number of particles in the PSO algorithm on the trade-off between power consumption and computation time is studied to have a good initialization. As the PSO algorithm also uses a maximum number of iterations for convergence criteria, we investigate a suitable initialization for this value. After this, the difference between the obtained associations using the different methods is highlighted. Lastly, the performance is evaluated and compared to conventional methods by varying the user QoS demand and cell radius. Note that only results with sufficient feasibility are considered.

The computational complexity of the MILP approach is evaluated in Fig. 2, where it can be seen that the number of candidate UAV positions increases the computation run time substantially. However, what is interesting to observe is that increasing the resolution of the quantized locations only leads to a marginal improvement (in fact only $0.6 \%$ ) in power consumption. The reason behind this is that the total network power consumption is dominated by the static power consumption of deploying a UAV. This result justifies the approach of using a quantized set of positions instead of the continuous 3D placement as it suggests the number of points can be relatively small.

Firstly, the proposed meta-heuristic PSO algorithm was fine-tuned for the purpose of simulation studies. The average power consumption and run time performance of the proposed algorithm were evaluated with a different number of particles. This is an important parameter as it ensures that the algorithm sufficiently explores the solution space. Figure 3 illustrates that increasing the number of particles beyond 18 offers a negligible reduction in power consumption while significantly increasing the run time.

The convergence behavior of the proposed PSO algorithm was also investigated. Figure 4 demonstrates how the relative power consumption is reduced per iteration. It was observed that after 50 iterations, the power consumption of more than $95 \%$ of realizations remained with a $1 \%$ margin of its optimal value. This is particularly relevant for setting the convergence criteria of the algorithm. It is worth mentioning that the drops in power correspond to the convergence of two UAV-SCs to the same location, which makes one of them redundant.

Figures 5 and 6 provide a comparison of the UAV-SC deployment association patterns obtained from the proposed methods. It is evident that the MILP method, which jointly optimizes the UAV-SC deployment, user associations, and power allocation, utilizes fewer UAV-SC and results in a slightly different association pattern to the PSO method.

The power consumption performance of both proposed methods was investigated further and compared to some existing techniques. Common strategies are to assume fixed 


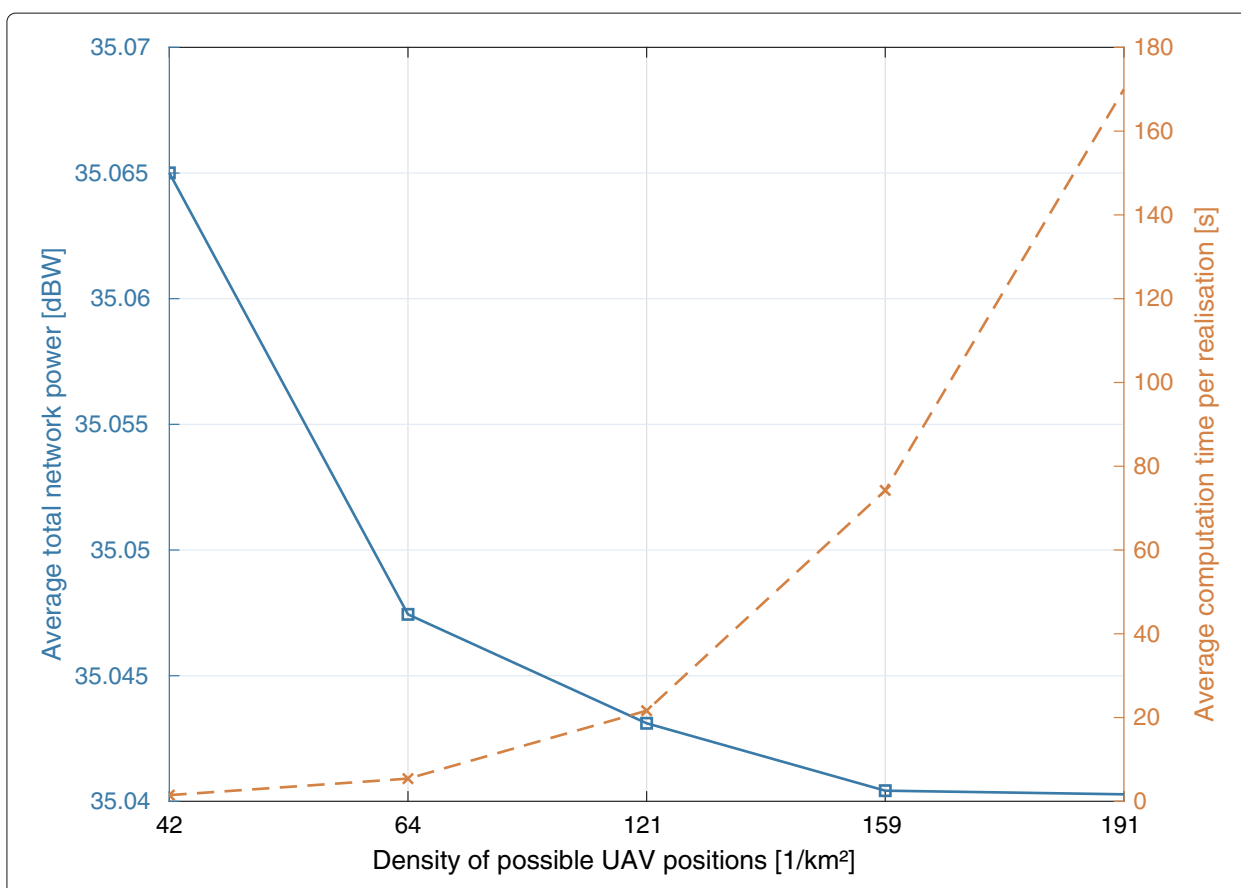

Fig. 2 Trade-off between power consumption and computation time with increasing quantization points

user association, based on distance and SNR, which are represented by "ASSOC_DIST" and "ASSOC_SNR." Many works assume the power allocation to always be fixed to maximum possible, regardless of the QoS constraint; this is shown as "FIX_POWER." Additionally, we also compare the performance of the proposed methods to an alternative meta-heuristic method known as simulated annealing, represented by "SA."

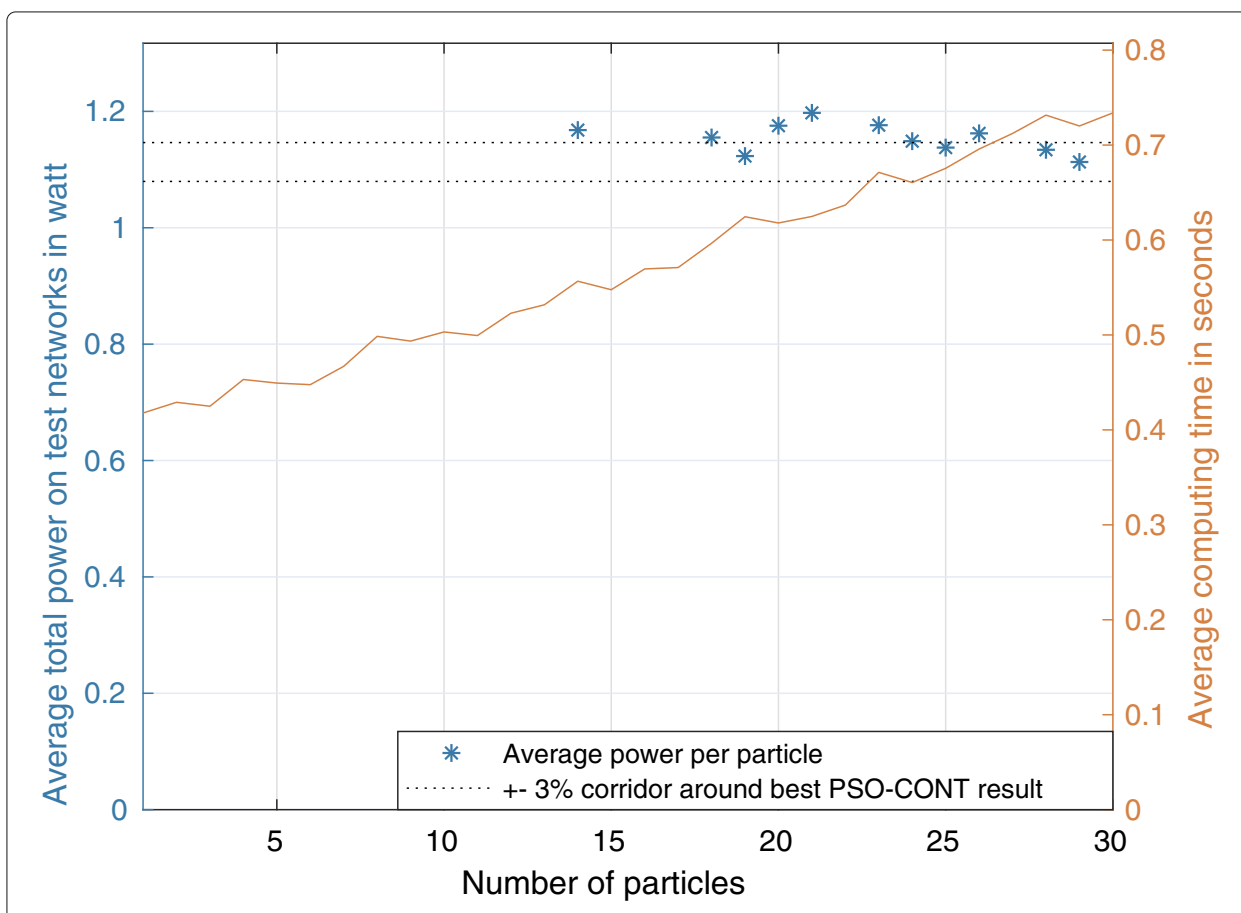

Fig. 3 Trade-off between power consumption and computation time with increasing number of particles 


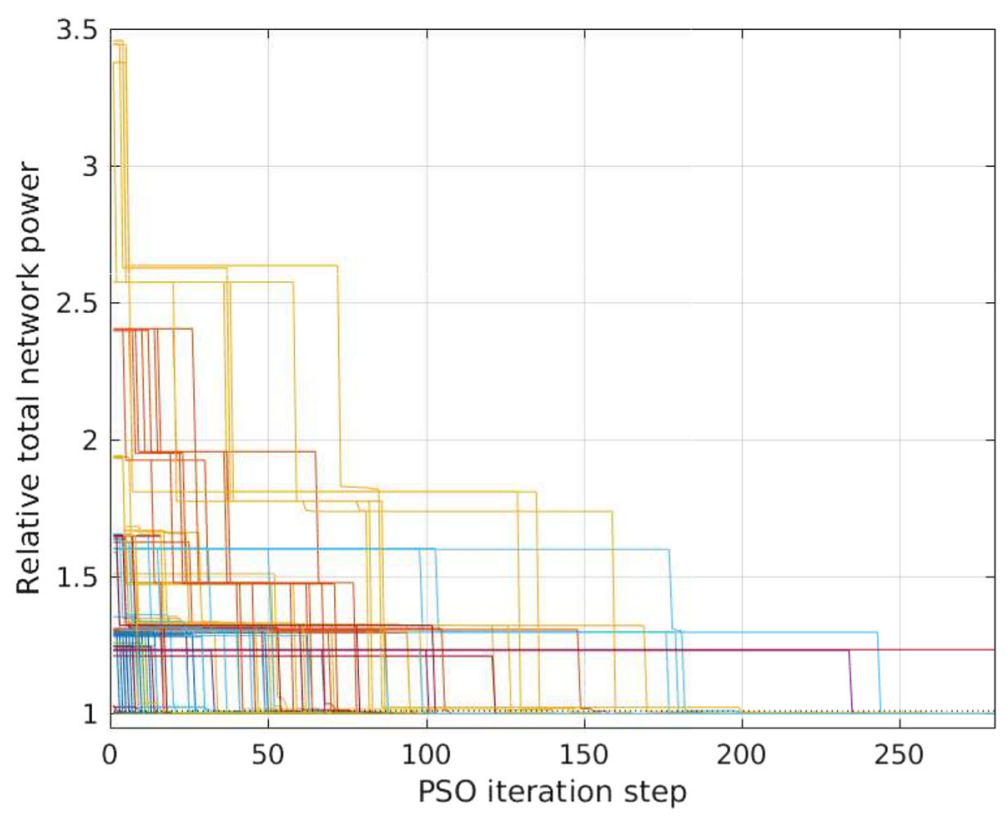

Fig. 4 Convergence pattern of the proposed PSO

The power consumption of the different methods is compared in Fig. 7. It is evident that the MILP method performs considerably better than the other techniques, achieving around $20 \%$ power reduction for lower SINR requirements and up to $10 \%$ for higher demands. Furthermore, it can be observed that the heuristic association schemes not only consume the most power but are also incapable of supporting high demands. This is due

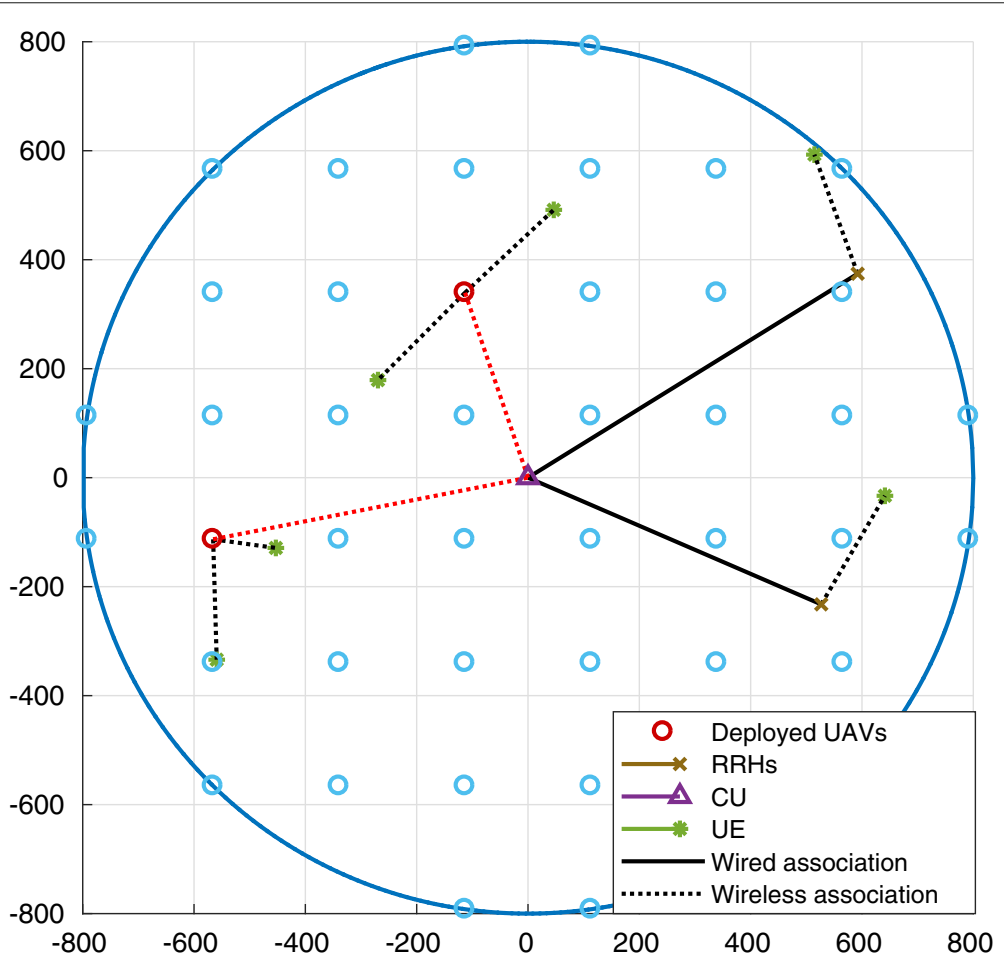

Fig. 5 Associations and UAV deployment obtained via the proposed MILP method 


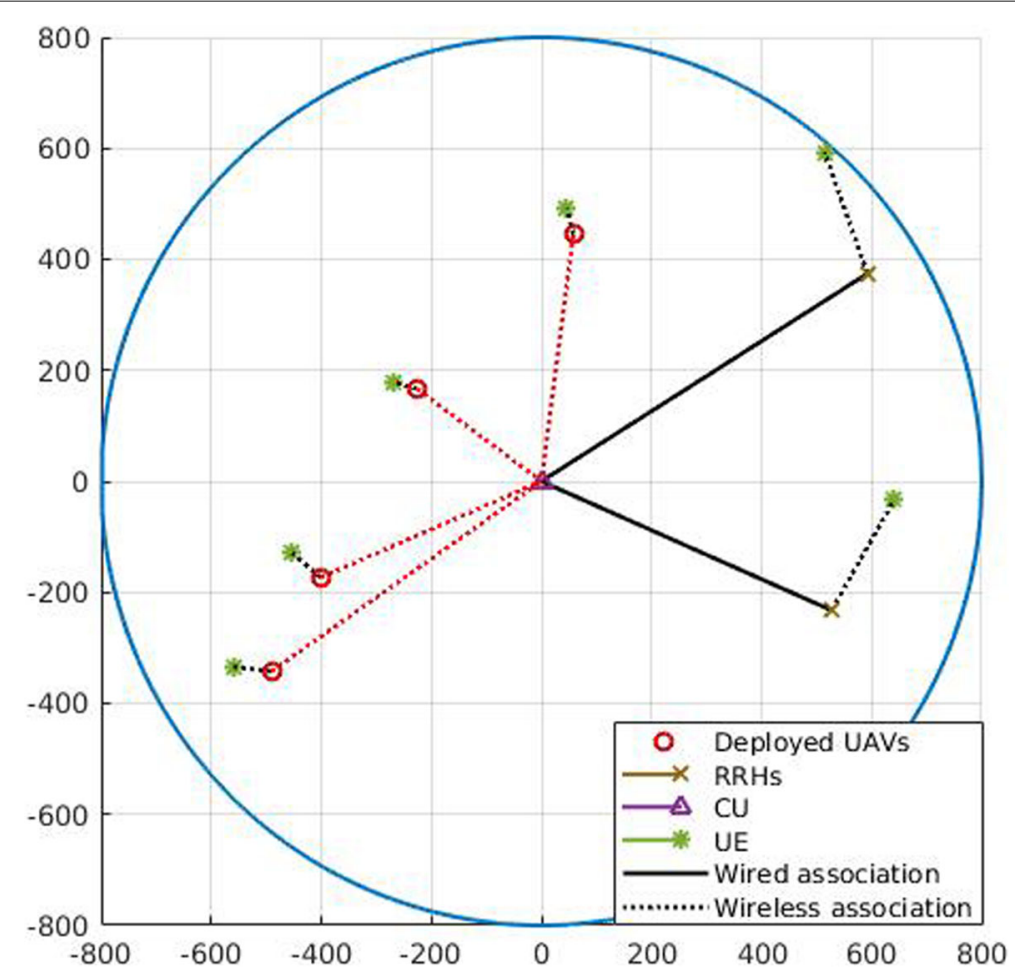

Fig. 6 Associations and UAV deployment obtained via the proposed PSO method

to the heavy interference experienced in these methods. A comparison of the number of deployed UAVs, as illustrated in Fig. 8, shows that the MILP method consistently utilizes the least number of UAV-SCs. Additionally, comparing the PSO and fixed power allocation scheme shows that although the PSO deployed slightly more number of UAV-SC, it still has a lower overall power consumption. This comparison highlights the gain in jointly optimizing the UAV-SC deployment position, user association, and power allocation.

The performance of the proposed techniques was also investigated with varying cell radius as shown in Fig. 9. This is particularly insightful as it affects the user density and the line of sights between UAV-SC and the users, and consequently the interference patterns. As the cell radius increases, the overall power consumption raises exponentially for all methods. However, the MILP-based solution leads to $15 \%$ less overall power consumption, when considering smaller cluster sizes and up to $8 \%$ for larger cells. Additionally, it is observed that even for a larger cell radius, the MILP and meta-heuristic methods are superior to fixed association and power allocation strategies. This is attributed to the deployment of fewer UAV-SC as well as a better power allocation as shown in Fig. 10.

We highlight that although the proposed PSO approach is inferior to the MILP, it benefits from a much lower computation time and therefore can be deemed suitable to larger size networks or perhaps used in real-time for dynamic network planning. Lastly, it is worth mentioning that the MILP approach was the only method that attained a $100 \%$ feasibility rate in the above simulation studies. Note that the feasibility rate is a measure of what percentage of the 200 realizations the different methods could solve without violating any constraints. 


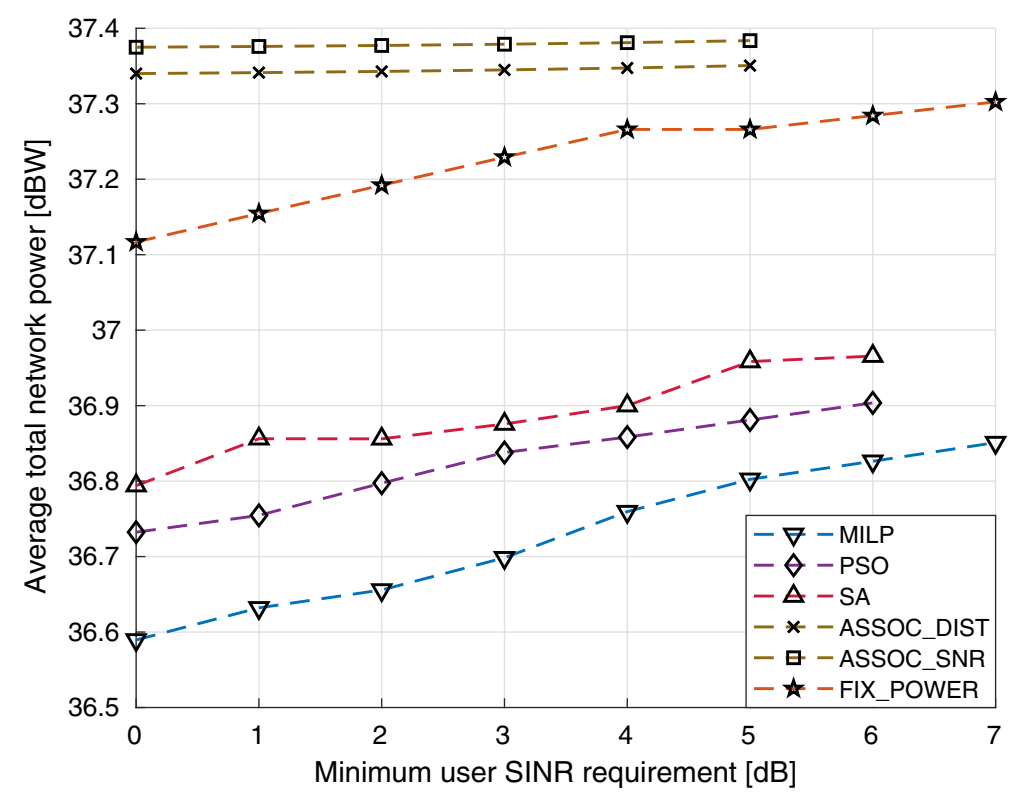

Fig. 7 Average power consumption with different schemes for varying minimum user QoS demand (missing points indicate infeasibility)

\section{Conclusion and future works}

In this paper, we considered the augmentation of a C-RAN with UAV-SC. We provided a design aiming at minimizing the overall power via jointly determining the number of UAV-SC, their hovering positions, associations, and the power allocation from the access points under to QoS, power, and fronthaul capacity constraints. As the original problem is an MINLP and computationally inefficient to solve, we provide two alternatives

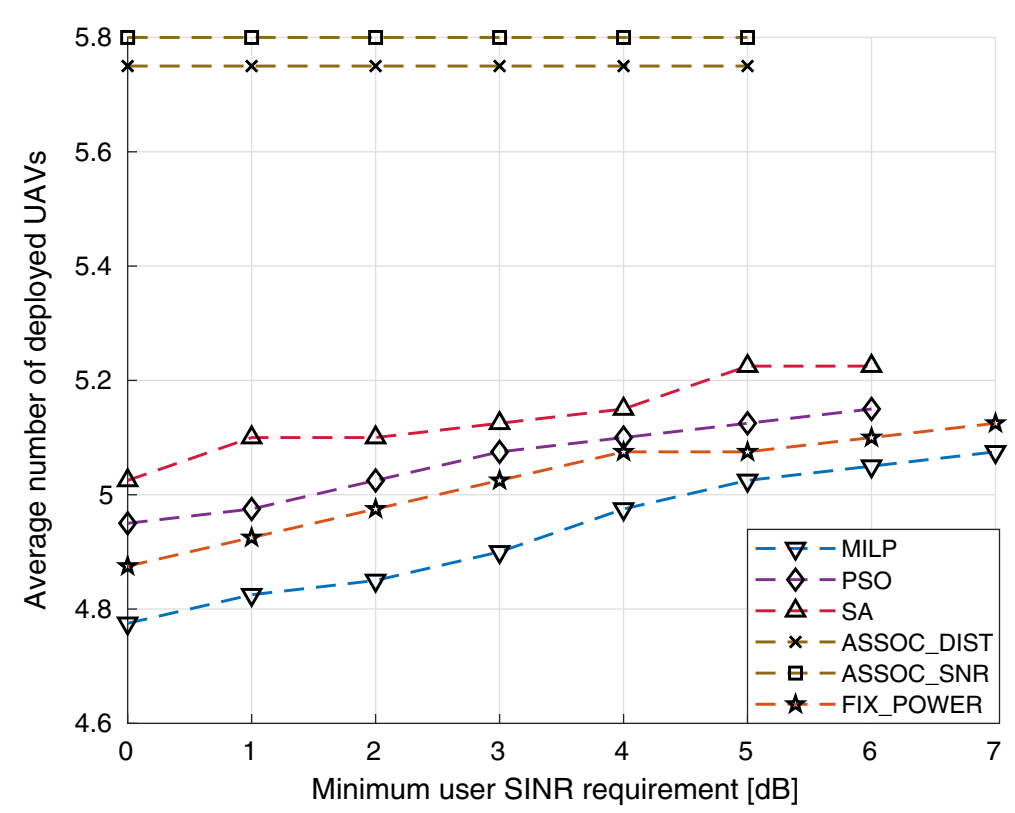

Fig. 8 Average number of UAVs deployed with different schemes for varying minimum user QoS demand (missing points indicate infeasibility) 


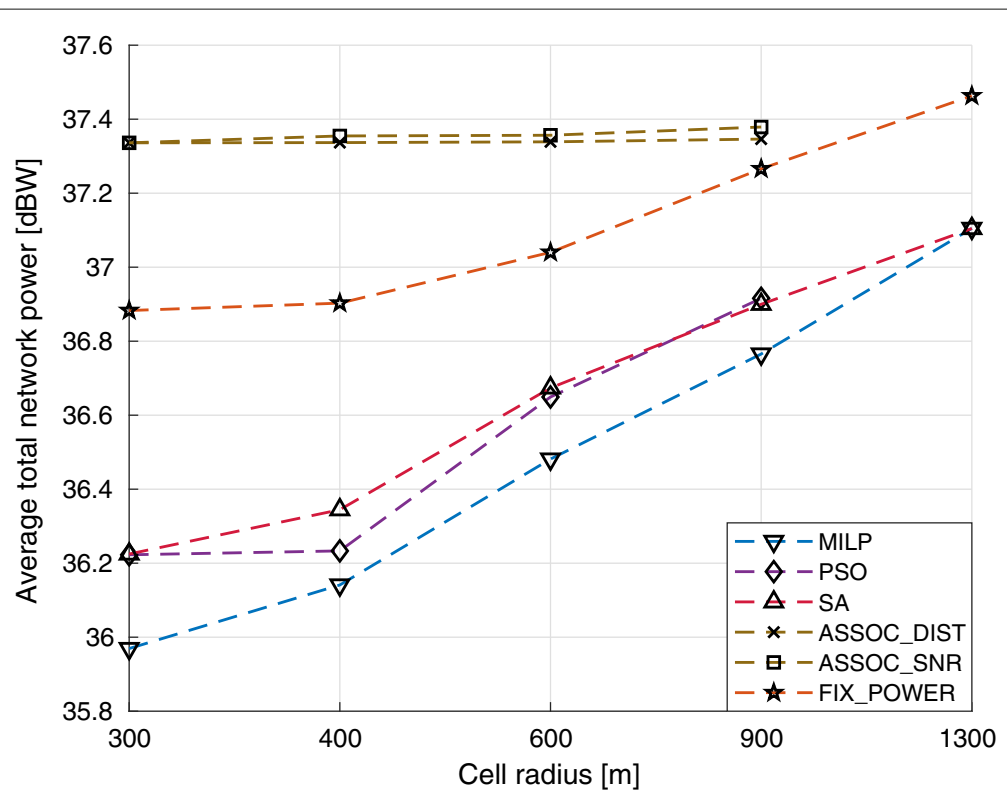

Fig. 9 Average power consumption with different schemes for varying cell radius (missing points indicate infeasibility)

approaching efficient solutions with relatively lower complexity. To this end, the original problem is reformulated as an MILP by quantizing the 3D UAV spatial placement, which can be solved efficiently via off-the-shelf solvers due to its linear structure. A faster meta-heuristic alternative is also proposed, which can be deemed suitable for larger size problems. Simulation studies suggest that the proposed methods consistently result in a fewer number of deployed UAV-SC and lower overall network power consumption,

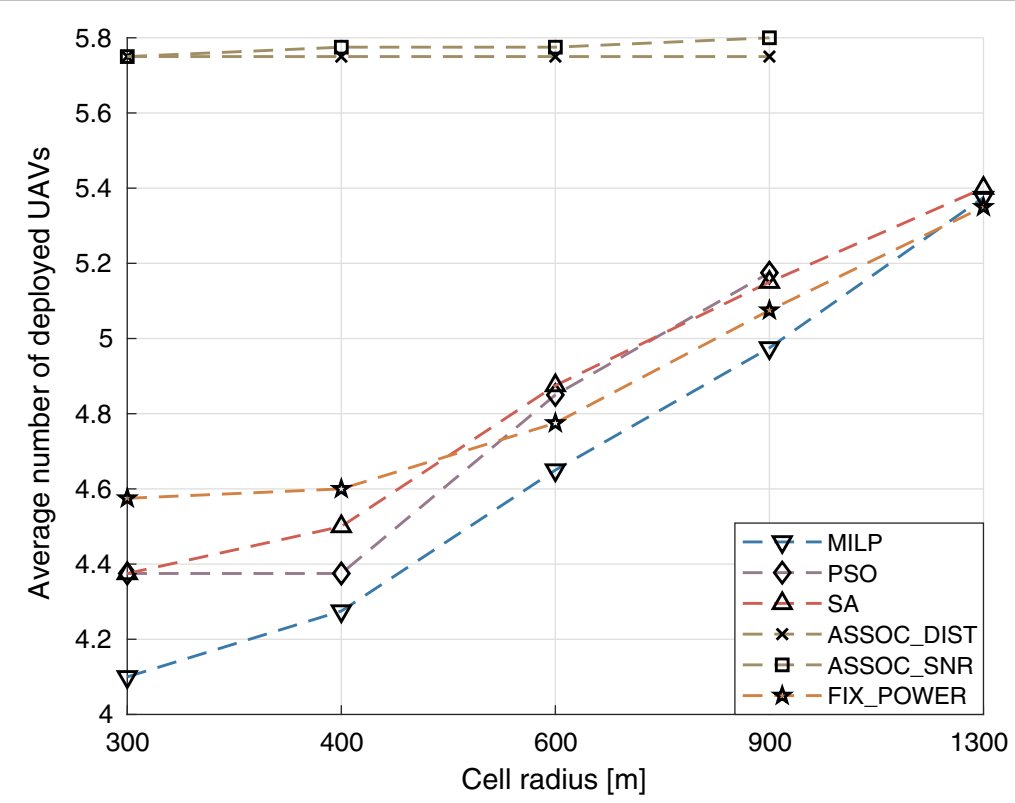

Fig. 10 Average number of UAVs deployed with different schemes for varying cell radius (missing points indicate infeasibility) 
in comparison to conventional techniques. In particular, considerable power savings are achieved for low QoS demands and dense scenarios.

\section{Methods/experimental}

The aim of performing simulation studies is to provide a numerical evaluation of the performance of the proposed methods and a comparison to conventional techniques. For this purpose, Monte Carlo simulations were performed, where channel realizations are generated according to Section 2 in MATLAB. The average power consumption is found by averaging the result of each realization over the total number of realizations and provided in watts. This provides a robust assessment of how the proposed techniques will perform under various network realizations. The infeasibility provides an average measure of how successful the method is in solving the different realizations. While the PSO algorithms were implemented from scratch, the optimization problems were solved using the free off-the-shelf solver, Gurobi.

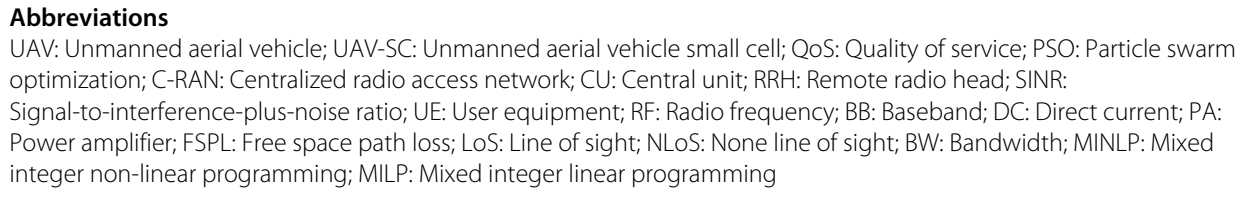

UAV: Unmanned aerial vehicle; UAV-SC: Unmanned aerial vehicle small cell; QoS: Quality of service; PSO: Particle swarm optimization; C-RAN: Centralized radio access network; CU: Central unit; RRH: Remote radio head; SINR:

Signal-to-interference-plus-noise ratio; UE: User equipment; RF: Radio frequency; BB: Baseband; DC: Direct current; PA: Power amplifier; FSPL: Free space path loss; LoS: Line of sight; NLoS: None line of sight; BW: Bandwidth; MINLP: Mixed integer non-linear programming; MILP: Mixed integer linear programming

\section{Authors' contributions}

All authors have contributed to this manuscript and approved the submitted manuscript.

\section{Funding}

The work in this paper has been supported by the German Research Council (DFG) within the basic research project DFG SCHM 2643/14-1 (Performance Analysis and Optimization of Future Low Latency and Reliable Cooperative

Communication). We gratefully acknowledge this support. Open Access funding enabled and organized by Projekt DEAL.

\section{Availability of data and materials}

The paper is self-contained. Simulation description and parameters are provided in details in Section 4.

\section{Competing interests}

The authors declare that they have no competing interests.

\section{Author details}

${ }^{1}$ ISEK Research Group, RWTH Aachen University, Kopernikusstr. 16, 52074, Aachen, Germany. ${ }^{2}$ Informationstheorie und Systematischer Entwurf von Kommunikationssystemen, RWTH Aachen University, Kopernikusstr. 16, 52074, Aachen, Germany.

Received: 24 April 2020 Accepted: 17 September 2020

Published online: 07 October 2020

\section{References}

1. A. Fotouhi, H. Qiang, M. Ding, M. Hassan, L. G. Giordano, A. Garcia-Rodriguez, J. Yuan, Survey on UAV cellular communications: practical aspects, standardization advancements, regulation, and security challenges. IEEE Commun. Surv. Tutor. 21(4), 3417-3442 (2019). https://doi.org/10.1109/COMST.2019.2906228

2. Z. Zhou, J. Feng, C. Zhang, Z. Chang, Y. Zhang, K. Huq, SAGECELL: Software-Defined Space-Air-Ground Integrated Moving Cells. IEEE Commun. Magazine. 56(8), 92-99 (2018)

3. Y. Liu, Z. Qin, Y. Cai, Y. Gao, G. Y. Li, A. Nallanathan, UAV communications based on non-orthogonal multiple access. IEEE Wirel. Commun. 26(1), 52-57 (2019). https://doi.org/10.1109/MWC.2018.1800196

4. M. Mozaffari, W. Saad, M. Bennis, Y. Nam, M. Debbah, A tutorial on UAVs for wireless networks: applications, challenges, and open problems. IEEE Commun. Surv. Tutor. 21(3), 2334-2360 (2019). http://arxiv.org/abs/1803.00680

5. Y. Li, L. Cai, UAV-assisted dynamic coverage in a heterogeneous cellular system. IEEE Network. 31(4), 56-61 (2017). https://doi.org/10.1109/MNET.2017.1600280

6. R. Fan, J. Cui, S. Jin, K. Yang, J. An, Optimal node placement and resource allocation for UAV relaying network. IEEE Commun. Lett. 22(4), 808-811 (2018). https://doi.org/10.1109/LCOMM.2018.2800737

7. J. Lyu, Y. Zeng, R. Zhang, T. J. Lim, Placement optimization of UAV-mounted mobile base stations. IEEE Commun. Lett. 21 (3), 604-607 (2016)

8. R. I. Bor-Yaliniz, A. El-Keyi, H. Yanikomeroglu, in IEEE International Conference on Communications (ICC), Efficient 3-D placement of an aerial base station in next generation cellular networks, (2016), pp. 1-5. https://doi.org/10.1109/ICC. 2016.7510820 
9. A. V. Savkin, H. Huang, Deployment of unmanned aerial vehicle base stations for optimal quality of coverage. IEEE Wirel. Commun. Lett. 8(1), 321-324 (2019). https://doi.org/10.1109/LWC.2018.2872547

10. M. Alzenad, A. El-Keyi, F. Lagum, H. Yanikomeroglu, 3-D placement of an unmanned aerial vehicle base station (UAV-BS) for energy-efficient maximal coverage. IEEE Wirel. Commun. Lett. 6(4), 434-437 (2017). https://doi.org/10 1109/LWC.2017.2700840

11. M. Mozaffari, W. Saad, M. Bennis, M. Debbah, Efficient deployment of multiple unmanned aerial vehicles for optimal wireless coverage. IEEE Commun. Lett. 20(8), 1647-1650 (2016). https://doi.org/10.1109/LCOMM.2016.2578312

12. P. Yang, X. Cao, X. Xi, Z. Xiao, D. Wu, Three-dimensional drone-cell deployment for congestion mitigation in cellular networks. IEEE Trans. Veh. Technol. 67(10), 9867-9881 (2018). https://doi.org/10.1109/TVT.2018.2857211

13. R. Ghanavi, E. Kalantari, M. Sabbaghian, H. Yanikomeroglu, A. Yongacoglu, in IEEE Wireless Communications and Networking Conference (WCNC), Efficient 3D aerial base station placement considering users mobility by reinforcement learning, (2018), pp. 1-6. https://doi.org/10.1109/WCNC.2018.8377340

14. J. He, J. Wang, H. Zhu, W. Cheng, P. Yue, X. Yi, in IEEE International Conference on Communications (ICC), Resource allocation in drone aided emergency communications, (2019), pp. 1-6. https://doi.org/10.1109/ICC.2019.8761897

15. E. Kalantari, I. Bor-Yaliniz, A. Yongaçoglu, H. Yanikomeroglu, User association and bandwidth allocation for terrestrial and aerial base stations with backhaul considerations, (2017), pp. 1-6. http://arxiv.org/abs/1709.07356

16. Z. Chang, W. Guo, X. Guo, T. Ristaniemi, Machine Learning-based Resource Allocation for Multi-UAV Communications System. IEEE ICC workshop (2020)

17. M. Mozaffari, W. Saad, M. Bennis, M. Debbah, in IEEE International Conference on Communications (ICC), Optimal transport theory for power-efficient deployment of unmanned aerial vehicles, (2016), pp. 1-6. https://doi.org/10. $1109 /$ ICC. 2016.7510870

18. H. Holtkamp, G. Auer, V. Giannini, H. Haas, A parameterized base station power model. IEEE Commun. Lett. 17(11), 2033-2035 (2013). https://doi.org/10.1109/LCOMM.2013.091213.131042

19. H. Ghazzai, M. Ben Ghorbel, A. Kadri, M. J. Hossain, H. Menouar, Energy-efficient management of unmanned aerial vehicles for underlay cognitive radio systems. IEEE Trans. Green Commun. Netw. 1(4), 434-443 (2017). https://doi. org/10.1109/TGCN.2017.2750721

20. S. Bi, J. Lyu, Z. Ding, R. Zhang, Engineering radio maps for wireless resource management. IEEE Wirel. Commun. 26(2), 133-141 (2019). https://doi.org/10.1109/MWC.2019.1800146

21. M. Clerc, J. Kennedy, The particle swarm-explosion, stability, and convergence in a multidimensional complex space. IEEE Trans. Evol. Comput. 6(1), 58-73 (2002). https://doi.org/10.1109/4235.985692

22. E. Kalantari, H. Yanikomeroglu, A. Yongacoglu, in IEEE 84th Vehicular Technology Conference (VTC-Fall), On the number and 3D placement of drone base stations in wireless cellular networks, (2016), pp. 1-6. https://doi.org/10.1109/ VTCFall.2016.7881122

23. I. Gurobi Optimization, Gurobi optimizer reference manual (2020). http://www.gurobi.com

24. E. Munapo, Solving the binary linear programming model in polynomial time, vol. 6, (2016), pp. 1-7

25. G. Yuan, B. Ghanem, Binary optimization via mathematical programming with equilibrium constraints. arXiv: Optimization and Control (2016)

26. L. T. H. An, T. P. Dinh, L. H. Minh, X. T. Vo, DC approximation approaches for sparse optimization. Eur. J. Oper. Res. 244, 26-46 (2015)

\section{Publisher's Note}

Springer Nature remains neutral with regard to jurisdictional claims in published maps and institutional affiliations.

\section{Submit your manuscript to a SpringerOpen ${ }^{\circ}$ journal and benefit from:}

- Convenient online submission

- Rigorous peer review

- Open access: articles freely available online

- High visibility within the field

- Retaining the copyright to your article

Submit your next manuscript at $\boldsymbol{\triangleright}$ springeropen.com 